\title{
Investigation of effect of design and operating parameters on acoustophoretic particle separation via 3D device-level simulations
}

\author{
Mehmet Akif Şahin ${ }^{1,2} \cdot$ Barbaros Çetin $^{3} \cdot$ M. Bülent Özer ${ }^{1,2}$
}

Received: 6 July 2019 / Accepted: 2 December 2019 / Published online: 16 December 2019

(c) Springer-Verlag GmbH Germany, part of Springer Nature 2019

\begin{abstract}
In the present study, a 3D device-level numerical model is implemented via finite element method to assess the effects of design and operating parameters on the separation performance of a microscale acoustofluidic device. Elastodynamic equations together with electromechanical coupling at the piezoelectric actuators for the stress field within the solid parts, Helmholtz equation for the acoustic field within fluid, and Navier-Stokes equations for the fluid flow are coupled for the simulations. Once the zero-acoustic and flow fields are obtained, the trajectories of the particles are obtained by employing point-particle approach. The particle trajectories are simulated for many particles with different sizes released from random initial locations. Separation performances of the different cases are evaluated based on described metrics such as purity, yield, percentage of particle stuck in the channel, the force acting on the particles, residence time and separation parameter.
\end{abstract}

Keywords Microfluidics $\cdot$ Particle manipulation $\cdot$ Acoustophoresis

\section{Introduction}

Among different techniques, acoustophoresis is a promising, label-free technique for bio-particle manipulation in microchannels with potential for high-throughput applications (Laurell et al. 2007; Cetin et al. 2014). Following the studies regarding the fundamental physics and demonstration of some successful applications prior to 2013 (Cetin et al. 2014), there has been even an increasing interest on microscale acoustofluidic technology for various biological, chemical and biomedical applications. Some recent studies include focusing of nanoparticles via a 2D acoustic field (Antfolk et al. 2014), removal of proteins from blood components (Tenje et al. 2015), precise rotational manipulation of single cells and organisms via streaming vortices (Ahmed et al. 2016), manipulation of micro-bodies (Kaynak

M. Bülent Özer

ozerb@metu.edu.tr; bulent.ozer@gmail.com

1 Mechanical Engineering Department, Middle East Technical University, 06800 Ankara, Turkey

2 Mechanical Engineering Department, TOBB University of Economics and Technology, 06560 Ankara, Turkey

3 Microfluidics and Lab-on-a-chip Research Group, Mechanical Engineering Department, İ.D. Bilkent University, 06800 Ankara, Turkey et al. 2017; Ceylan et al. 2017), separation of nanoparticles in a continuous flow (Sehgal and Kirby 2017; Mengxi et al. 2017), enrichment of mononuclear cells from blood (Urbansky et al. 2017), clinical scale enrichment of tumor cells from blood for either higher tumor cell recovery or higher purity (Magnusson et al. 2017), activation of sensory neurons in C. elegans for neurobiology research (Zhou et al. 2017), microfluidic cell sorting at comparable rate and purity levels of commercial cell sorters (Ung et al. 2017), rapid hematocrit measurement of blood with a readout accuracy of 3\% points hematocrit (Petersson et al. 2018), purification of lymphocytes from leukopheresis product, buffy coat and whole blood (Lissandrello et al. 2018), separation of bacteria from blood at high throughput as well as at high cell concentration with a bacterial yield as high as $99.7 \%$ (Ohlsson et al. 2018), isolation of three clinically relevant bacteria from blood with improved limit of detection (Silva et al. 2017; Dow et al. 2018), and high-throughput platelet separation with a recovery rate as high as $87 \%$ with a plastic microfluidic device (Gu et al. 2019). Even though many successful cases have been demonstrated in the literature, efficiency of acoustophoretic bio-particle separation may be low since the selectivity of acoustophoresis relies on the size and acoustic properties of bio-particles which may not vary significantly among different bio-particle populations. To increase selectivity and hence improve separation efficiency, 
integration of acoustophoresis with dielectrophoresis (Cetin et al. 2016; Antfolk et al. 2017) and labeling bio-particles with negative acoustic contrast particles (Cushing et al. 2018) have also been proposed in the literature.

The essential ingredient for acoustophoretic particle manipulation is acoustic radiation force which acts on a particle as a result of scattering of acoustic waves at the particle-fluid interface. Acoustic radiation force is a secondorder time averaged effect. Considering the second-order perturbations on pressure and density field, the acoustic radiation force on a spherical particle derived analytically at the Rayleigh limit (i.e. by recognizing only single scattering event) which requires size of the particle being much smaller than the wavelength of the incoming acoustic wave in an inviscid fluid (King 1934; Yosioka and Kawasima 1955; Gorkov 1962). Later, the derivation was extended to include viscous (Settnes and Bruus 2012) and thermoviscous effects (Karlsen and Bruus 2015). The presence of multiple particles and/or microchannel confinement dictates inclusion of re-scattering events to constitute acoustic particle-particle (Wang and Dual 2009; Silva and Bruus 2014; Baasch et al. 2017) and particle-wall (Wang and Dual 2012) interactions. With more sophisticated numerical simulations, acoustic radiation force can be obtained for non-spherical particles (Glynne-Jones et al. 2013; Hahn et al. 2015; Garbin et al. 2015) and/or for cylindrical (Cai et al. 2010) and spherical particles beyond Rayleigh limit (Cai et al. 2010; Baasch and Dual 2018).

Device-level simulations are also as crucial as determination of acoustic radiation force to understand the effects of design and operating parameters on the device performance. For a complete picture, the solution of elastodynamic equations are required for the solid parts together with electromechanical coupling at the piezoelectric actuators as well as full compressible Navier-Stokes equations for the fluid flow. Moreover, a rigorous model needs to account for the presence of the particles. Device-level simulations are challenging owing to multi-scale and coupled multi-physics nature of the problem. There are different time scales (acoustic cycle in the range of sub-microseconds, transient behavior of the device in the range of miliseconds and particle motion in the range of seconds) and different length scales (size of viscous and thermal boundary layers in the range of sub-micrometer, size of a particle in the range of tens of micrometers, size of microchannels in the range of hundred micrometers and size of a device in the range of centimeters) are involved in the problem. The time scale problem can be overcome by employing time-harmonic approach (Hahn et al. 2014). To overcome the multiple length scales, it is a common approach to treat particles as point particles and use predefined relations for calculation of the acoustic radiation and drag forces acting on the particles. For this approach, simulations are performed without the presence of particles within the computational domain. Even without the particles, simulations of all the remaining length scales are not feasible, especially for 3D problems. Several groups carried out device-level simulations including all length scales in 2D (Muller et al. 2012, 2013; Nama et al. 2015; Liu et al. 2016; Mao et al. 2016). On the other hand, many 3D simulations studied the fluid flow and particle trajectories without considering the dynamics of piezoelectric actuators and solid parts of the device (Trippa et al. 2011; Gralinski et al. 2012; Trujillo et al. 2013; Buyukkocak et al. 2014; Yang et al. 2018; Vitali et al. 2018). In these studies, the mode shapes of the piezoelectric actuator were not modeled and the calculation of the acoustic radiation force relied on 1D transient acoustic field which assumes uniform distribution of boundary displacement on the channel wall. A uniform distribution of microchannel wall displacement along the microchannel axis is an idealization which cannot be realized in practice owing to the (1) complex mode shapes along the axial direction experienced by the piezoelectric actuator at high frequencies, (2) possible inhomogeneities within the piezoelectric actuators and (3) effect of real microchannel architecture on the acoustic field. Moreover, an ad hoc parameter (which is not know a priori) to account for the acoustic energy provided by the piezoelectric actuator needs to be introduced into the 1D models (Trippa et al. 2011; Gralinski et al. 2012; Trujillo et al. 2013; Buyukkocak et al. 2014; Yang et al. 2018; Vitali et al. 2018). For a realistic picture, 3D device-level simulations which couples the dynamics of the piezoelectric material to the acoustic response are essential (Hahn et al. 2014; Cetin et al. 2016). In addition, the effect of the viscosity can be further incorporated into 3D device-level simulations through the concept of limit velocity without any calculations within the viscous boundary layer (Lei et al. 2014; Hahn et al. 2014, 2015). Furthermore, certain variability in particle size, acoustic properties and/or release locations also needs to be considered in the model to mimic real acoustophoretic applications (Yang et al. 2018; Vitali et al. 2018; Garofalo Jun 2019; Buyukkocak et al. 2014; Cetin et al. 2016).

\subsection{Scope of the present study}

A schematic drawing of a typical acoustophoretic device based on bulk acoustic waves is given in Fig. 1. All the parameters, which can be classified as design parameters shown on the figure, are important for a successful operation of an acoustophoretic chip. In addition to design parameters, there are also operating parameters (namely frequencies and amplitude of the actuation signal and volumetric flow rates) which also affect the device performance. The effects of these parameters may be assessed thoroughly employing a device-level computational model. Moreover, a Monte Carlo type of approach (Buyukkocak et al. 2014; Cetin et al. 2016) which provides 


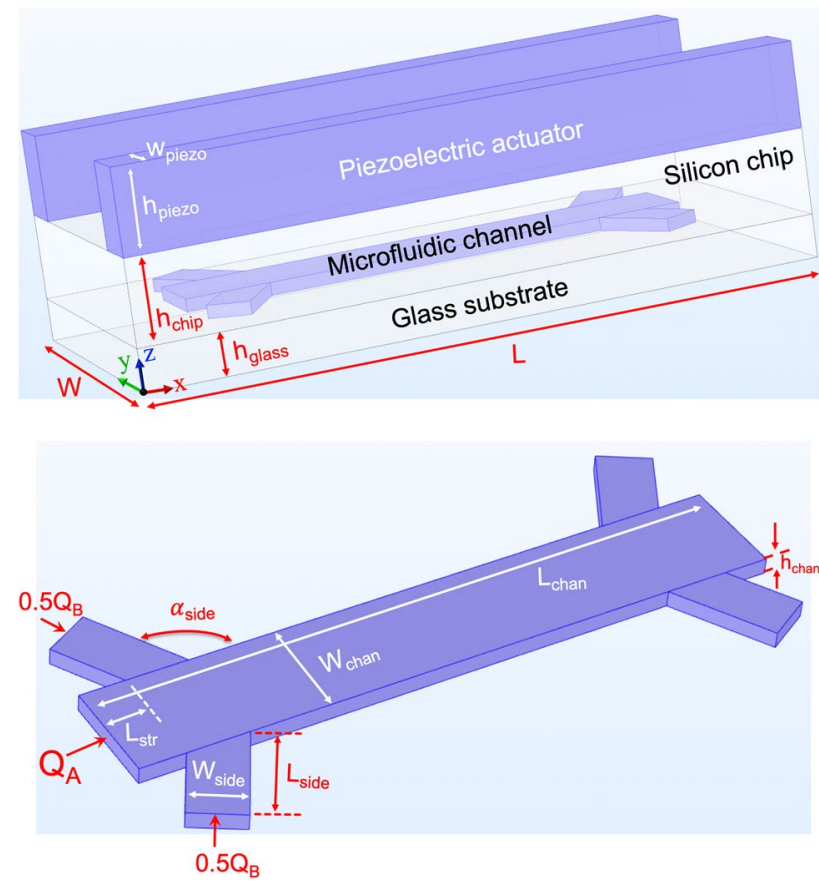

Fig. 1 Schematics of the computational domain

statistical distributions for the starting locations of particles as well as size distribution is employed for more realistic modeling. In the present study, a 3D device-level numerical model is implemented via COMSOL Multiphysics to assess the effects of design and operating parameters on the separation performance of a microscale acoustofluidic device. The particle trajectories are obtained for 660 particles with 2 different size groups released from random initial locations. The simulations are performed for different channel widths, frequencies, microchannel depths and volumetric flow rates. Some metrics (such as purity, yield, percentage of particle stuck in the channel, force acting on the particles, residence time and separation parameter) have been introduced to discuss the separation performance of the device. To the best of authors' knowledge, a critical assessment of design and operating parameters via 3D device-level simulations which couples the dynamics of the piezoelectric material to the acoustic response is not available in the literature, and we believe understanding the sensitivity of these parameters on the separation performance will be benefited by the researchers in the microfluidics community for further development of bulk acoustic wave acoustophoretic devices.

\section{Computational model}

The acoustophoretic system analyzed in this study is composed of a glass substrate, a microfluidic channel network etched onto a silicon material, two piezoelectric actuators and a buffer fluid filling the microfluidic channel. The piezoelectric materials actuate the chip which has a channel pattern on the bottom side of the chip. A glass lid covers the microchannel from the bottom. The geometric parameters are shown in Fig. 1.

The physics of the problem governs with elastodynamic equations for the solid parts (i.e. the microfluidic chip, substrate and piezoelectric actuators) together with electromechanical coupling at the piezoelectric actuators and with Navier-Stokes equations for the fluid flow. Fluid-solid coupling interfaces are present between the solid and fluid domains. The device is excited by a timeharmonic signal applied to the piezoelectric actuator. The problem can be well treated by a perturbation expansion. The time-harmonic fields can be written as

$\mathbf{u}(\mathbf{x}, t)=\mathbf{u}(\mathbf{x}) \mathrm{e}^{-j \omega t}$,
$\mathbf{v}_{1}(\mathbf{x}, t)=\mathbf{v}_{1}(\mathbf{x}) \mathrm{e}^{-j \omega t}$,
$\rho_{1}(\mathbf{x}, t)=\rho_{1}(\mathbf{x}) \mathrm{e}^{-j \omega t}$,

$p_{1}(\mathbf{x}, t)=p_{1}(\mathbf{x}) \mathrm{e}^{-j \omega t}=c_{f}^{2} \rho_{1}(\mathbf{x}) \mathrm{e}^{-j \omega t}$,

where $\mathbf{u}(\mathbf{x})$ is the displacement field in the structural part of the device. $\mathbf{v}_{1}(\mathbf{x})$ is the first-order velocity, $\rho_{1}(\mathbf{x})$ is the first-order density, and $p_{1}(\mathbf{x})$ is the first-order pressure fields within the fluidic domain.

The time-harmonic elastodynamic equation for the solid parts reads as

$\nabla \cdot \mathbf{T}=-\rho \omega^{2} \mathbf{u}$,

where $\mathbf{T}$ is the stress field, $\rho$ is the material density. For the chip material and substrate, a linear elastic model is implemented which reads the stress-strain relation as

$\mathbf{T}_{i j}=-\lambda \delta_{i j} \mathbf{S}_{k k}+2 G \mathbf{S}_{i j}=\mathbf{C}_{E} \cdot \mathbf{S}$,

where $\mathbf{S}$ is the strain field, $\mathbf{C}_{E}$ is the elasticity matrix. $\lambda$ is the Lamé's first parameter, $G$ is the shear modulus which are related to Poisson's ratio and Young's modulus as

$\lambda=\frac{E v}{(1+v)(1-2 v)}, \quad G=\frac{E}{2(1+v)}$.

Kinematics relates the strain and displacement fields as

$\mathbf{S}=\frac{1}{2}\left[(\nabla \mathbf{u})^{T}+\nabla \mathbf{u}\right]$.

Modeling of piezoelectric actuator requires an electromechanical coupling, hence dependences of the stress and displacement fields on the strain and electric fields need to be 
defined. The linear constitutive relations for the piezoelectric actuator can be written as

$\mathbf{T}=\widetilde{\mathbf{C}}_{E} \cdot \mathbf{S}-\mathbf{e}^{t r} \cdot \mathbf{E}$,

$\mathbf{D}=\mathbf{e} \cdot \mathbf{S}+\tilde{\boldsymbol{\varepsilon}} \cdot \mathbf{E}$,

where $\mathbf{e}$ is the piezoelectric coupling matrix, $\mathbf{D}$ is the electric displacement field, and $\mathbf{E}$ is the electric field. $\widetilde{\mathbf{C}}_{E}$ is the elasticity and $\tilde{\boldsymbol{\varepsilon}}$ is the dielectric matrices which are complex quantities defined as

$\widetilde{\mathbf{C}}_{E}=\mathbf{C}_{E}\left[1-j \eta_{S}\right]$,

$\tilde{\boldsymbol{\varepsilon}}=\boldsymbol{\varepsilon}\left[1-j \eta_{\varepsilon S}\right]$,

where $\eta_{S}$ is the isotropic structural loss coefficient, $\eta_{\varepsilon S}$ is the dielectric loss factor which account for mechanical damping and dielectric loss, respectively. Equation (11) can be also used in conjunction with Eq. (6) to take into account the structural damping for the linear elastic materials.

The charge conservation in terms of electric displacement field reads as

$\nabla \cdot \mathbf{D}=0$.

First-order pressure field in a quiescent fluid can be written as

$\nabla^{2} p_{1}=-k^{2} p_{1}, \quad k=(1+j \gamma) \frac{\omega}{c_{f}}, \quad \gamma=\frac{(1+\beta) \mu \omega}{2 \rho_{0} c_{f}^{2}}$,

where $p(\mathbf{x})$ is the first-order pressure field, $c_{f}$ is the speed of sound in the fluid and $\omega$ is the angular frequency, $\gamma$ is the viscous damping factor, $\beta$ is the viscosity ratio, $\mu$ is the viscosity of fluid, $\rho_{0}$ is the density of the quiescent fluid. In microfluidics, typically flow speeds are low (i.e. $\left|\mathbf{v}_{o}\right| \leq 0.1 \mathrm{~m} / \mathrm{s}$ ), where $\mathbf{v}_{o}$ is the zero-order velocity field); therefore, the effect of the zero-order velocity on the first-order velocity and pressure fields can be ignored, and Eq. (14) can still be employed for the first-order pressure field (Bruus 2011). Considering the operation at room temperature together with a frequency in the order of $\mathrm{MHz}$, the viscous damping factor

The zero-order flow field is governed by the continuity and Stokes equation due to low Reynolds number nature of the flow field:

$\nabla \cdot \mathbf{v}_{0}=0$,

$-\nabla p_{0}+\mu \nabla^{2} \mathbf{v}_{0}=0$.

Following the perturbation analysis for higher order terms, second-order continuity and Navier-Stokes equations can be derived (Bruus 2012a). Normally, the second-order terms are negligible compared to the first-order ones. However, time-harmonic nature of the first-order fields contributes to the time-averaged second-order effects. Therefore, time-averaged second-order equations are employed for the acoustophoretic applications. Second-order effects are responsible for acoustic streaming through which a fluid motion is induced by the acoustic waves. The thin viscous boundary layer in the first-order fields near the channel walls needs to be resolved to take acoustic streaming for the fluid motion into account, which makes the model computationally extremely expensive especially for 3D simulations. The length scale of the viscous boundary layer is $\delta=\sqrt{2 \mu / \rho_{0} \omega}$, which is sub-micrometer for water at frequencies in the order of $\mathrm{MHz}$ at room temperature (Settnes and Bruus 2012). Although there are some alternative ways are available to include acoustic streaming as a boundary condition for the zero-order velocity field based on semi-analytical treatment of the flow physics inside the boundary layer (at least for microchannels without any sharp edges or tips) (Lei et al. 2014; Hahn et al. 2015), considering the flow rate values, particle and channel sizes investigated in this study, we neglect the effect of acoustic streaming on the fluid flow and particle trajectories.

The second-order effects are also responsible for acoustic radiation force acting on the particles. Although it is a second-order effect, together with the inviscid flow approximation which is valid when the particle radius is much greater than the viscous boundary layer thickness (i.e. $a \gg \delta$ ), the acoustic radiation force can be calculated based on time-average of the first-order quantities as Bruus (2012b)

$\mathbf{F}_{\text {rad }}=-\int_{\mathcal{S}_{0}}\left[\left(\frac{1}{2 \rho_{0} c_{f}^{2}}\left\langle p_{1}^{2}\right\rangle-\frac{1}{2} \rho_{0}\left\langle\mathbf{v}_{1}^{2}\right\rangle\right) \mathbf{n}+\rho_{0}\left\langle\left(\mathbf{n} \cdot \mathbf{v}_{1}\right) \mathbf{v}_{1}\right\rangle\right] d \mathcal{S}$,

for water can be approximated as $10^{-6}$, therefore, the complex valued wavenumber $k$ can be replaced by a real valued wavenumber $k_{0}=\omega / c_{f}$ in Eq. (14) (Bruus 2012a). where $\mathcal{S}_{0}$ is either the particle surface or any surface encompassing the particle. $p_{1}$ and $\mathbf{v}_{1}$ terms have contributions both from the incident and scattering components (i.e. 
$\left.p_{1}=p_{1, \text { in }}+p_{1, s c}, \mathbf{v}_{1}=\mathbf{v}_{1, \text { in }}+\mathbf{v}_{1, s c}\right)$. The incident components $\left(p_{1, \text { in }}^{2}, \mathbf{v}_{1, \text { in }}^{2}\right)$ will cancel out each other during the integration over the control surface (Glynne-Jones et al. 2013). Strictly speaking, the first-order field needs to be obtained with the presence of the particle, followed by the integration. Although some special treatment may be required for the calculation of the integral depending on size of the particle (Glynne-Jones et al. 2013), when the particle size is small compared to the channel dimensions, Rayleigh limit (i.e. single scattering events) is valid and any particle-wall and particle-particle acoustic interactions can be ignored (Baasch and Dual 2018). Consequently, the acoustic radiation force can be written in terms of radiation potential (Gorkov 1962):

$\mathbf{F}_{\mathrm{rad}}=-\nabla U_{\mathrm{rad}}$,

$U_{\mathrm{rad}}=\frac{4 \pi}{3} a^{3}\left[f_{1} \frac{1}{2 \rho_{f} c_{f}^{2}}\left\langle p_{\text {in }}^{2}\right\rangle-f_{2} \frac{3}{4} \rho_{f}\left\langle\mathbf{v}_{\text {in }}^{2}\right\rangle\right]$,

$f_{1}=1-\frac{\rho_{0} c_{f}^{2}}{\rho_{\mathrm{p}} c_{\mathrm{p}}^{2}}, \quad f_{2}=\frac{2\left(\rho_{\mathrm{p}}-\rho_{0}\right)}{2 \rho_{\mathrm{p}}+\rho_{0}}$.

where $\rho_{\mathrm{p}}$ is the density of the particle, $c_{\mathrm{p}}$ is the speed of sound of the particle, $f_{1}$ is the monopole coefficient, and $f_{2}$ is the dipole coefficient. In the equations above, the pressure and velocities are for incident waves which are equal to the first-order pressure and velocity fields without the presence of the particles. The monopole and dipole coefficients introduce the effect of the scattered waves from the particle. In the inviscid limit, the relation between the first-order pressure and velocity fields reads as

$\mathbf{v}_{1}=-\frac{j}{\rho_{0} \omega} \nabla p_{1}$.

Although the inviscid assumption can be relieved and the monopole expression can be corrected for viscous effects, since we consider only polystyrene particles whose density is very close to that of water, and hence buoyant, there is no need for any correction for viscous effects in this study (Settnes and Bruus 2012). Once the zero-order and firstorder pressure and flow fields are obtained, the trajectory of the particles can be obtained by employing point-particle approach (i.e. Lagrangian tracking method Cetin et al. 2017). Newton's second law of motion can be written as

$m_{\mathrm{p}} \frac{d \mathbf{v}_{\mathrm{p}}}{\mathrm{d} t}=\mathbf{F}_{\mathrm{rad}}+\mathbf{F}_{\mathrm{drag}}+\mathbf{F}_{\mathrm{g}}$, where $m_{p}$ is the mass of the particle, $\mathbf{v}_{\mathrm{p}}$ is the particle velocity, $\mathbf{F}_{\text {drag }}$ is the drag force and $\mathbf{F}_{\mathrm{g}}$ is the gravity force. The model is built on COMSOL Multiphysics and material properties are assigned using the material library of the COMSOL ("silicon" for the microfluidic chip material, "silica glass" for the substrate, "Lead Zirconate Titanate (PZT4)" for the piezoelectric actuator and "water" for the fluid medium). Due to the low Reynolds number nature of the simulated cases, Stokes flow is employed for the drag force calculations. In addition, in COMSOL, predefined wall corrections that take into account hydrodynamic particle-wall interactions for the calculation of the drag forces are also included in some case studies and no significant change in the results is observed. The simulations for each case typically take about $15-20$ minutes on a 6-cores i7-8750h processors at $2.20 \mathrm{GHz}$ and with $32 \mathrm{~GB}$ of RAM.

\section{Performance metrics}

Two groups of particles with different diameters (small and large particles) are used for the simulations of each case. Three different diameters are simulated: $5 \mu \mathrm{m}, 8 \mu \mathrm{m}$ and $15 \mu \mathrm{m}$. All the particles are made of polystyrene. Total number of 660 particles (330 small particles +330 large particles) are released for each case in groups of 60 particles from randomly distributed positions from the side inlets to mimic the experimental conditions (probability of a particle releasing from any point on the cross-section is the same). The following nomenclature for defining the size and exit locations of the particles is used in the performance metrics:

(1) Count_ $\langle A\rangle_{-}\langle\mathrm{B}\rangle$ : Number of $\langle\mathrm{B}\rangle$ sized particles exiting from the $\langle A\rangle$ exit or stuck in the channel $\langle A\rangle$ is either 'main', 'side' or 'stuck', and $\langle\mathrm{B}\rangle$ is either 'small' or 'large'.

(2) Count_Total_ $\langle A\rangle$ : Number of $\langle A\rangle$ sized particles in the simulation. $\langle A\rangle$ is either 'small' or 'large'.

(3) $\mu_{\langle\mathrm{A}\rangle}^{\langle\mathrm{B}\rangle}$ : Mean value of $y$-position of $\langle\mathrm{A}\rangle$ sized particles at the $\langle\mathrm{B}\rangle$ region of the channel $\langle\mathrm{A}\rangle$ is either 'small' or 'large', and $\langle\mathrm{B}\rangle$ is either 'lower' or 'upper'.

(4) $\sigma_{\langle\mathrm{A}\rangle}^{\langle\mathrm{B}\rangle}$ : Standard deviation of $y$-position of $\langle\mathrm{A}\rangle$ sized particles at the $\langle\mathrm{B}\rangle$ region of the channel $\langle\mathrm{A}\rangle$ is either 'small' or 'large', and $\langle\mathrm{B}\rangle$ is either 'lower' or 'upper'.

Five parameters are introduced to discuss the separation performance of the device, as follows. 


\subsection{Purity}

The main exit channel is intended for large particles to exit and side exit channels are intended for small particles. Therefore, purity is defined as follows:

Purity $_{\text {main }}=\frac{\text { Count_main_large }}{\text { Count_main_large }+ \text { Count_main_small }}$,

Purity $_{\text {side }}=\frac{\text { Count_side_small }}{\text { Count_side_large }+ \text { Count_side_small }}$.

\subsection{Yield}

Yield is the measure of what percentage of initially released large or small particles are recaptured at their intended exit locations:

Yield $_{\text {large }}=\frac{\text { Count_main_large }}{\text { Count_Total_large }}$,

Yield $_{\text {small }}=\frac{\text { Count_side_small }}{\text { Count_Total_small }}$.

Yield may be low due to two reasons, either particles got stuck in the channel and did not leave the channel from any of the exits or they exited from the unintended channel.

\section{$3.3 \%$ Stuck}

\% Stuck denotes the percent of particles that did not exit from any of the separation channel exits during the simulation.

$\%$ Stuck $_{\text {large }}=\frac{\text { Count_stuck_large }}{\text { Count_Total_large }}$,

$\%$ Stuck $_{\text {small }}=\frac{\text { Count_stuck_small }}{\text { Count_Total_small }}$.

The occurrence of stuck particles generally is due to pressure gradients occurring in directions other than the aimed separation direction. For instance, if there exist pressure gradients in the entry side channels then the particles may get stuck in the entry region even before entering the main channel. Moreover, it is possible to have pressure gradient especially in the height directions (mostly occurs when the depth of the channel is comparable to the wavelength of the acoustic waves) which leads to particles moving very close to the top and bottom walls of the channel. Since the velocity is small at the vicinity of the top and the bottom walls due to the no-slip boundary condition, the reduced drag force cannot carry the particles against the acoustic radiation force, hence particles which are either trapped or moving very slowly are counted as stuck particles.

\subsection{Average force}

The average acoustophoretic force on a single particle means acoustophoretic force experienced by each particle until the time they exit the separation channel or until the time they get stuck at a wall. Average of this acoustophoretic force across all the particles with large diameter or small diameter gives the average acoustophoretic force for large or small diameter particles.

\subsection{Separation metric}

The separation metric is intended for quantification of separation performance. A check point location is defined just before the side channel separation junction (please see Fig. 2). The $x$ - and $y$-locations of each particle are stored in the simulation for each time step. Each particle's $y$-location is stored as they pass the check point location. The mean value of $y$-locations of large and small particles as they pass the check point is evaluated at the upper and the lower separation regions. Standard deviations of $y$-locations at the separation point for large and small particles are also evaluated at upper and lower separation regions. The schematic representation of the separation metric is shown in Fig. 2. A similar performance parameter as separation efficiency was also recently introduced for acoustophoretic separation in the literature (Yang et al. 2018; Garofalo Jun 2019).

$(\text { Separation Metric })_{\text {upper }}=\frac{\mu_{\text {large }}^{\text {upper }}-\mu_{\text {small }}^{\text {upper }}}{\left(\sigma_{\text {large }}^{\text {upper }}+\sigma_{\text {small }}^{\text {upper }}\right) / 2}$,

$(\text { Separation Metric })_{\text {lower }}=\frac{\mu_{\text {large }}^{\text {lower }}-\mu_{\text {small }}^{\text {lower }}}{\left(\sigma_{\text {large }}^{\text {lower }}+\sigma_{\text {small }}^{\text {lower }}\right) / 2}$,

Separation Metric

$$
=\frac{(\text { Separation Metric })_{\text {upper }}+(\text { Separation Metric })_{\text {lower }}}{2} .
$$

\subsection{Mean residence time}

Residence time for a particle is the amount of time that passes between entering the channel from a side entry and leaving the channel from a side exit or the main exit. The 
Fig. 2 Schematic representation of separation metric

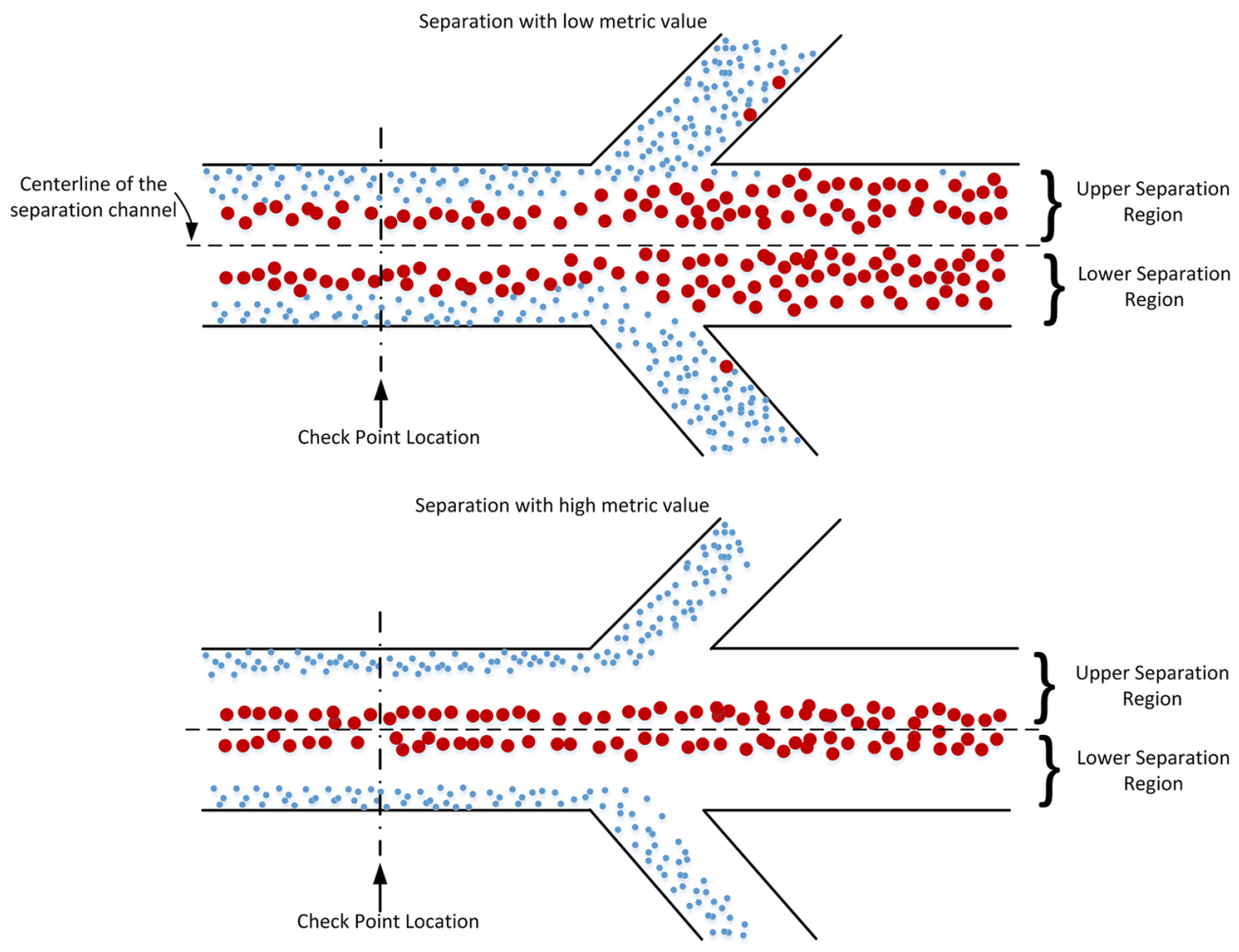

average of all residence times for all the particles which exit the separation channel is the mean residence time.

\section{Results and discussion}

The geometric parameters for the acoustophoretic chip may be different for chips with different resonant frequencies. The geometric parameters are shown in Fig. 1. All simulation parameters are tabulated in Table 2. The mesh for each simulation is selected to ensure at least ten elements for the simulated wavelength. In the numerical studies, nominal flow rates (if the flow rate is not changed to investigate the effect) are $10 \mu \mathrm{L} / \mathrm{min}$ for each side channel (microparticle flow rate) and $60 \mu \mathrm{L} / \mathrm{min}$ for the main entry (buffer flow rate) which is a total flow rate of 80 $\mu \mathrm{L} / \mathrm{min}$. Simulations are performed at different voltage levels and the value of the voltage that results in the highest purity and yield values is chosen as the excitation voltage for the case study.

\subsection{Separation with $1.0 \mathrm{~mm}$ channel width (Case-1)}

In Case-1, separation of $5 \mu \mathrm{m}$ and $15 \mu \mathrm{m}$ particles are demonstrated. The channel width is $1.0 \mathrm{~mm}$ which is the resonant channel width for acoustophoretic force. The resonance frequency of the piezoelectric material along the thickness direction is around $700 \mathrm{kHz}(722 \mathrm{kHz})$. Figure 3 shows the performance parameters of the separation together with the distribution of particles at the outlets within the microchannel. It can be seen from the top plot that purity at the channel outlets for $5 \mu \mathrm{m}$ and $15 \mu \mathrm{m}$ particles are high meaning that the most of the particles exited from the intended outlets they are supposed to. Yield values are around $80 \%$, and $15 \mu \mathrm{m}$ particles are separated with slightly higher yield. The yield values are lower than the purity values due to the stuck particles inside the channel. Average acoustophoretic forces acting on the $5 \mu \mathrm{m}$ and $15 \mu \mathrm{m}$ particles are calculated, and the average force is in the order of picoN and the average force on the large particles is almost 30 times higher than the average force on the small particles. The average amount of time that particles spent in the separation chamber is $1.3 \mathrm{~s}$. Separation metric is calculated to be 4.2 (the metric value will be more informative when compared to other cases).

\subsection{Separation with different channel widths}

\subsubsection{At the corresponding resonance frequencies (Case-2)}

In Case-2, separation of $5 \mu \mathrm{m}$ and $15 \mu \mathrm{m}$ particles is demonstrated for different channel widths. The piezoelectric actuators are excited at the corresponding resonant frequencies. Channel widths of $330 \mu \mathrm{m}, 660 \mu \mathrm{m}, 1.0 \mathrm{~mm}$ and $1.65 \mathrm{~mm}$ which corresponds to piezoelectric actuator thicknesses of $1.0 \mathrm{~mm}, 2.0 \mathrm{~mm}, 3.0 \mathrm{~mm}$ and $5.0 \mathrm{~mm}$ are simulated. These thickness values correspond to resonance frequencies around 2.0 MHz, $1.0 \mathrm{MHz}, 700 \mathrm{kHz}$ and $400 \mathrm{kHz}$, respectively (see 
Table 1 Simulation parameters

\begin{tabular}{|c|c|c|c|}
\hline \multicolumn{4}{|c|}{ Geometric parameters (referring to Fig 1) } \\
\hline $\begin{array}{l}\text { Length of computational } \\
\text { domain }\end{array}$ & $L$ & 8.0 & {$[\mathrm{~mm}]$} \\
\hline $\begin{array}{l}\text { Width of computational } \\
\text { domain }\end{array}$ & $W$ & 4.0 & {$[\mathrm{~mm}]$} \\
\hline Thickness of glass substrate & $h_{\text {glass }}$ & 0.5 & {$[\mathrm{~mm}]$} \\
\hline Thickness of chip & $h_{\text {chip }}$ & 1.0 & {$[\mathrm{~mm}]$} \\
\hline $\begin{array}{l}\text { Thickness of piezoelectric } \\
\text { actuator }\end{array}$ & $h_{\text {piezo }}$ & $1.0,2.0,3.0,5.0$ & {$[\mathrm{~mm}]$} \\
\hline $\begin{array}{l}\text { Width of piezoelectric } \\
\text { actuator }\end{array}$ & $W_{\text {piezo }}$ & $\mathrm{W} / 6$ & \\
\hline Length of channel & $L_{\text {chan }}$ & 6.0 & {$[\mathrm{~mm}]$} \\
\hline Height of channel & $h_{\text {chan }}$ & $0.15,0.25$ & {$[\mathrm{~mm}]$} \\
\hline Width of main channel & $W_{\text {chan }}$ & $0.33,0.66,1.0,1.5$ & {$[\mathrm{~mm}]$} \\
\hline Width of side channel & $W_{\text {side }}$ & $W_{\text {chan }} / 2$ & \\
\hline Angle for side channel & $\alpha_{\text {side }}$ & $120^{\circ}$ & \\
\hline Diameter of particles & $d_{p}$ & $5.0,8.0,15$ & {$[\mu \mathrm{m}]$} \\
\hline \multicolumn{4}{|l|}{ Operating Parameters } \\
\hline Flow rate at main channel & $Q_{A}$ & $10,20,30,60$ & {$[\mu \mathrm{L} / \mathrm{min}]$} \\
\hline Flow rate at side channels & $Q_{B}$ & 10 & {$[\mu \mathrm{L} / \mathrm{min}]$} \\
\hline Frequency of applied voltage & $f$ & $0.40 .7,1.0,2.0$ & {$[\mathrm{MHz}]$} \\
\hline \multicolumn{4}{|l|}{ Polystyrene } \\
\hline Density & $\rho_{p}$ & 1050 & {$\left[\mathrm{~kg} / \mathrm{m}^{3}\right]$} \\
\hline Speed of sound & $c_{p}$ & 2300 & {$[\mathrm{~m} / \mathrm{s}]$} \\
\hline \multicolumn{4}{|l|}{ Silicon } \\
\hline $\begin{array}{l}\text { Isotropic structural loss coef- } \\
\text { ficient }\end{array}$ & $\eta_{S}$ & $1 \times 10^{-4}$ & \\
\hline \multicolumn{4}{|l|}{ Glass } \\
\hline $\begin{array}{l}\text { Isotropic structural loss coef- } \\
\text { ficient }\end{array}$ & $\eta_{S}$ & $1 \times 10^{-3}$ & \\
\hline
\end{tabular}

The material properties are taken from COMSOL Material Library at $T=20^{\circ}$ unless otherwise stated

Table 1 for the dimensions). The purity and yield of particle separation at these frequencies are illustrated in Fig. 4a. It can be observed that the purity and yield values are similar for the chips designed for the resonance frequencies of around $2 \mathrm{MHz}, 1 \mathrm{MHz}$ and $700 \mathrm{kHz}$. The separation purity and yield significantly drops for the chip designed to resonate around $400 \mathrm{kHz}$.

As shown in Fig. $4 \mathrm{~b}$, the number of stuck particles increases as the acoustic resonance frequency of the separation channel decreases. The chip which resonates at 400 $\mathrm{kHz}$ has a channel width of $1.65 \mathrm{~mm}$ whereas the chip with the $2 \mathrm{MHz}$ resonance frequency has a channel width of 0.33 $\mathrm{mm}$ with the same channel depth for both chips. Since the flow rate values in the simulations are the same for all of the chips (regardless of the resonance frequency of the chip), the particles in the $400 \mathrm{kHz}$ chip flow in the largest crosssectional area, therefore, they have the lowest flow velocity. This fact exhibits itself as increased residence time in the chips with lower resonance frequency (see Fig. 4d). In addition to the acoustic force in the separation direction, particles also experience forces along the height direction of the channels (with smaller amplitudes compared to forcing amplitudes in separation direction). When the particles move with small velocities and reside longer times in low resonance frequency channels, they have more time to move towards the top and bottom walls of the channel where the flow velocity is small, and hence the drag force acting on the particles is small. Therefore, the particles are either trapped or moving very slowly which increases the number of stuck particles at lower resonance frequency chips. In addition, Fig. $4 \mathrm{c}$ shows that the particles need higher acoustophoretic forces for separation as the separation frequency increases. High-frequency resonance chips have smaller channel widths and particles flow with higher velocities, therefore, they need higher acoustophoretic force for a successful separation in shorter time. Average acoustophoretic forces acting on the $5 \mu \mathrm{m}$ and $15 \mu \mathrm{m}$ particles are calculated, and the average force is in the order of picoN and the average force on the large particles is almost 30 times higher than that of the smaller particles. This value is slightly different than what the theory predicts (27 times) since each particle takes a different trajectory, therefore, experiencing different acoustic pressures and forces during their motion. The theoretical ratio would be observed if all the particles were experiencing the same acoustic pressures during their motion. Figure $4 \mathrm{~d}$ represents that as the chip resonance frequency increases, average residence time for the particles increases. This is due to fact that high-resonance frequency chips have smaller channel widths. Figure $4 \mathrm{~d}$ also shows that the separation metric is the highest in the $1.0 \mathrm{MHz}$ chip and it is significantly lower for $400 \mathrm{kHz}$ chip. Figure $4 \mathrm{e}$ shows the particle distributions of a low separation metric value (at the inlet and outlet of a $400 \mathrm{kHz}$ chip) and a high separation metric value (at the inlet and outlet of a $700 \mathrm{kHz}$ chip). It can be seen from the lower part of Figure $4 \mathrm{e}$ that $700 \mathrm{kHz}$ chip has a better separation than that of the $400 \mathrm{kHz}$ chip as interpreted by the separation metric.

\subsubsection{At the same frequency (case-3)}

In Case-3, different channel widths of $330 \mu \mathrm{m}, 660 \mu \mathrm{m}, 1.0$ $\mathrm{mm}$ and $1.65 \mathrm{~mm}$ are used for particle separation, however different from Case-2, the piezoelectric actuators with a thickness of $5.0 \mathrm{~mm}$ are excited at a fixed frequency of 400 $\mathrm{kHz}$. The purpose of Case- 3 is to evaluate the possibility of separating the particles even if the width of the channel is not equal to half of the wavelength of the pressure wave in the fluid. Figure 5a shows the purity and yield of particle separation at different channel widths when everything else is kept the same, namely the geometric properties of the chip (chip width, length, thickness) as 
Fig. 3 Separation and performance metrics in $1.0 \mathrm{~mm}$ channel with $700 \mathrm{kHz}$ resonance: a separation of $5 \mu \mathrm{m}$ and $15 \mu \mathrm{m}$ particles, $\mathbf{b}$ purity and yield, $\mathbf{c}$ $\%$ stuck and force, $\mathbf{d}$ separation metric and residence time

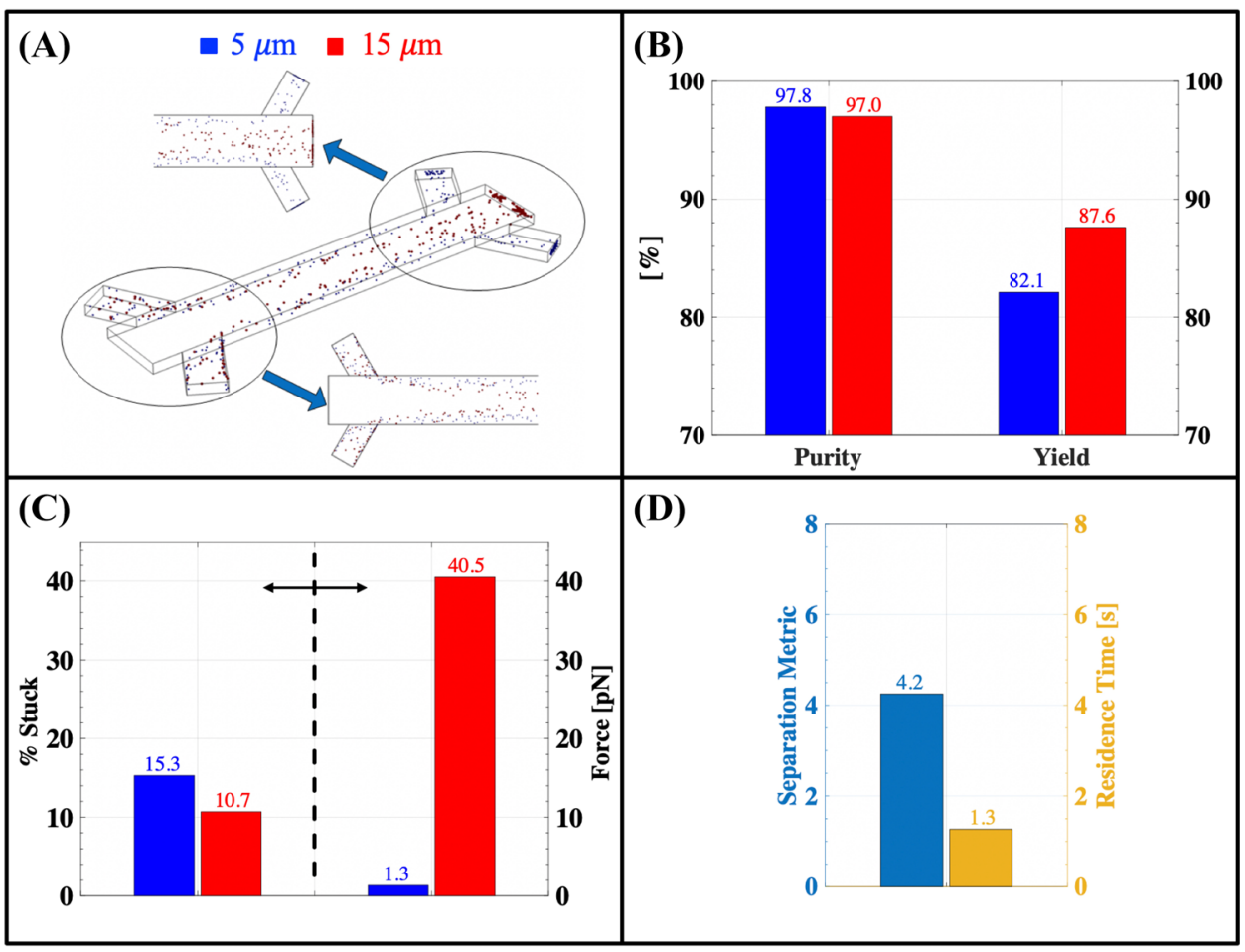

well as the excitation and geometric variables of the piezoelectric actuators (dimensions, frequency). As seen from the figure, successful separation of microparticles is possible for all channel widths. $660 \mu \mathrm{m}$ channel width seems to be performing better than the separation in other channel widths. Figure $5 \mathrm{~b}$ illustrates the number of particles which are stuck in the channel. It is clear that the number of stuck particles is the lowest when the channel width is $1.65 \mathrm{~mm}$ (which is the closest channel width to the half wavelength at the excitation frequency of $400 \mathrm{kHz}$ ). Figure 6 illustrates how the acoustic pressure distributions look like for each channel width. Similar distribution patterns and values are observed for each chip with different channel widths. Furthermore, the pressure amplitudes inside the separation channels are also similar. However, since the acoustic forces are related to spatial gradients of pressure, higher acoustic forces are generated in channels with smaller width. Additionally, the required voltages are also provided to reach the required acoustic pressures in the caption of Fig. 6. Since $1.65 \mathrm{~mm}$ channel width is the closest value for the half wavelength of the acoustic pressure wave at $400 \mathrm{kHz}$, the voltage is the lowest for that channel width. For the smaller channel widths, the required voltages are almost an order of magnitude higher than the voltages required in Case-2. For instance, in Case-2, the voltage required for separation in a chip with $1.0 \mathrm{~mm}$ channel width is $3 \mathrm{~V}$ at $722 \mathrm{kHz}$; however, if the separation is simulated in the same chip but with piezoelectric actuators operating at $400 \mathrm{kHz}$ (Case-3), the required voltage becomes $120 \mathrm{~V}$. These higher voltages at small channel widths make the separation practically impossible due to heating issues which certainly exhibit themselves at these high voltages.

\subsection{Separation at different channel depths (case-4)}

In Case-4, different channel depths of $50 \mu \mathrm{m}, 150 \mu \mathrm{m}$, $250 \mu \mathrm{m}, 500 \mu \mathrm{m}, 750 \mu \mathrm{m}$ and $950 \mu \mathrm{m}$ are used. The channel width used in this study is $1.0 \mathrm{~mm}$ which corresponds to a resonance frequency around $700 \mathrm{kHz}$. The purpose of Case- 3 is to evaluate the effect of channel depth in acoustophoretic separation. In all channel depths, the side and main inlet flow rates are the same. Figure 7 a shows the purity and yield of $5 \mu \mathrm{m}$ and $15 \mu \mathrm{m}$ particles. $250 \mu \mathrm{m}$ depth seem to be resulting in favorable outcomes in terms of purity and yield. Lower purity and yield results are obtained when the depth of the channel is about half the wavelength of the acoustic pressure wave $(950 \mu \mathrm{m})$ at $700 \mathrm{kHz}$. Figure $7 \mathrm{~b}$ illustrates the acoustic force required decreases with increasing depth since the velocity of the particles drop as the channel depth gets larger (since the same flow rate is applied for all channel depths). The percentage of particles stuck in the channel diminshes as the channel depths increase (sedimentation under gravity is not modeled). The reason for this low number of stuck particles in deeper channels is possibly due to the low acoustic forces on particles. Even though the number of stuck particles is small for deep channels, it is clear from Fig. 7a that the purity and yield are 
Fig. 4 Performance metrics and separation at different resonance frequencies: a purity and yield, b $\%$ stuck, $\mathbf{c}$ average acoustophoretic force, $\mathbf{d}$ separation metric and residence time, $\mathbf{e}$ separation of $5 \mu \mathrm{m}$ and $15 \mu \mathrm{m}$ particles
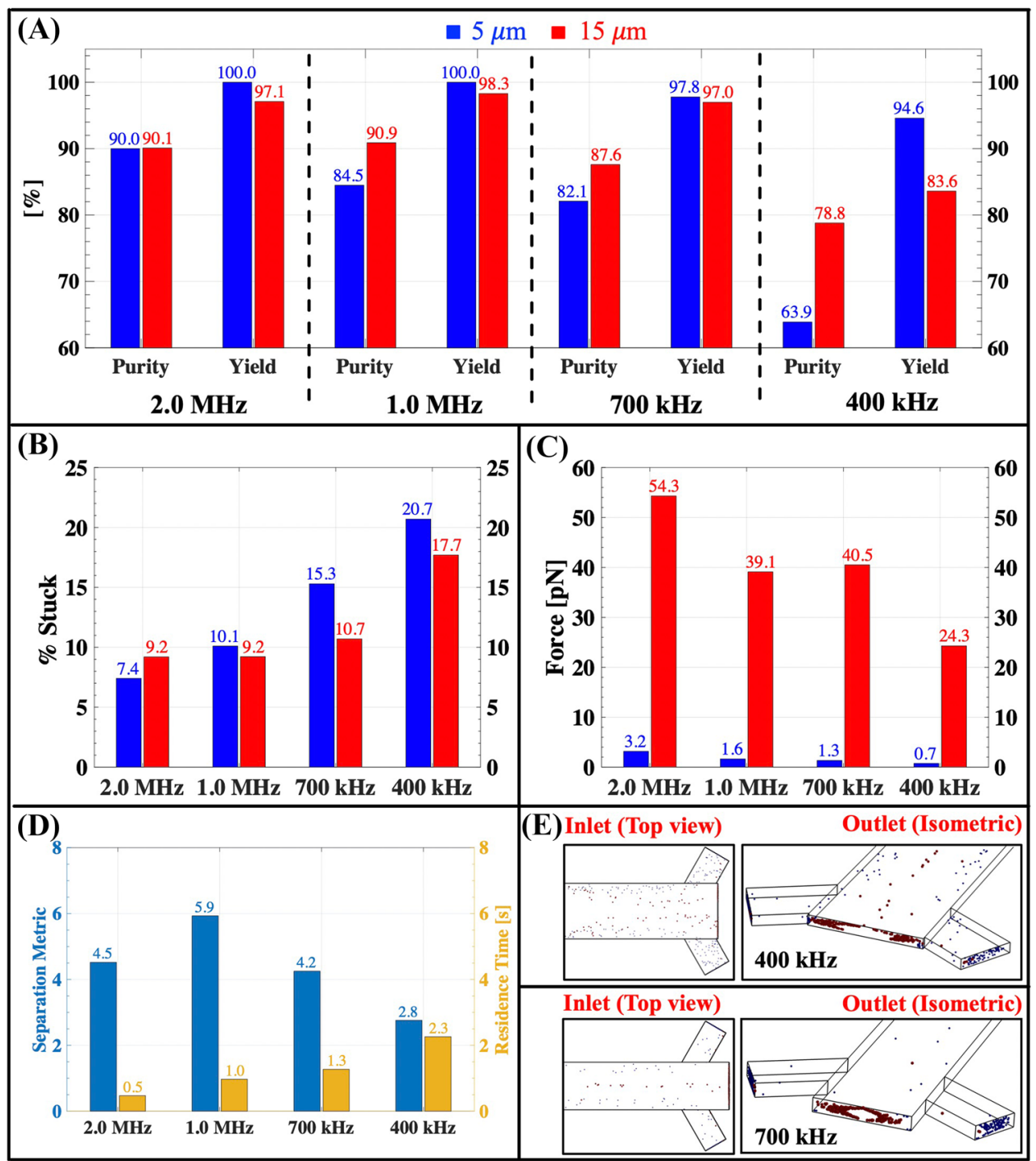

low for deep channels. The separation metric demonstrated in Fig. $7 \mathrm{~d}$ also favors $250 \mu \mathrm{m}$ depth channel for successful separation of $5 \mu \mathrm{m}$ and $15 \mu \mathrm{m}$ particles. Figure $7 \mathrm{~d}$ depicts a trend that as the residence time inside the channel increases, the separation metric decreases. Figure 7e shows the particle distributions at the main and side outlets. It can be observed that for $250 \mu \mathrm{m}$ depth channel, particles seem to exit the channels almost uniformly from all channel depths. Moreover, in $250 \mu \mathrm{m}$ channel depth chip, $5 \mu \mathrm{m}$ and $15 \mu \mathrm{m}$ particles are clearly separated from each other. On the other hand, for the channel with a depth of $950 \mu \mathrm{m}$, the particles experience clear movement along the height of the channel since the depth is comparable to the wavelength. As seen from the figure, the red particles moved to the bottom of the channel resulting in inefficient separation. The blue particles can be seen at the unintended main channel exit and the red particles can be seen exiting from the unintended side channel exits. The existence of both size particles in all exits makes the separation process difficult. If the amplitude of the voltage is increased, the blue particles will move towards the main channel (unintended channel); on the other hand, if the voltage is decreased, then the red particles will move towards the side channels (unintended channel). Therefore, it is not possible to improve separation performance through voltage adjustments of the piezoelectric actuators for the $950 \mu \mathrm{m}$ depth channel.

\subsection{Separation with different flow rate ratios (case-5)}

In Case-5, the flow rates of the main and side channels are arranged such that particles coming from side inlets stay close to the walls of the separation chamber. The channel width is $1.0 \mathrm{~mm}$ which corresponds to $700 \mathrm{kHz}$ thickness resonance condition of the PZTs. The performance of separation is evaluated for the main flow to sheath flow 
Fig. 5 Performance metrics at different channel widths: a purity and yield, $\mathbf{b} \%$ stuck, $\mathbf{c}$ average acoustophoretic force, $\mathbf{d}$ separation metric and residence time

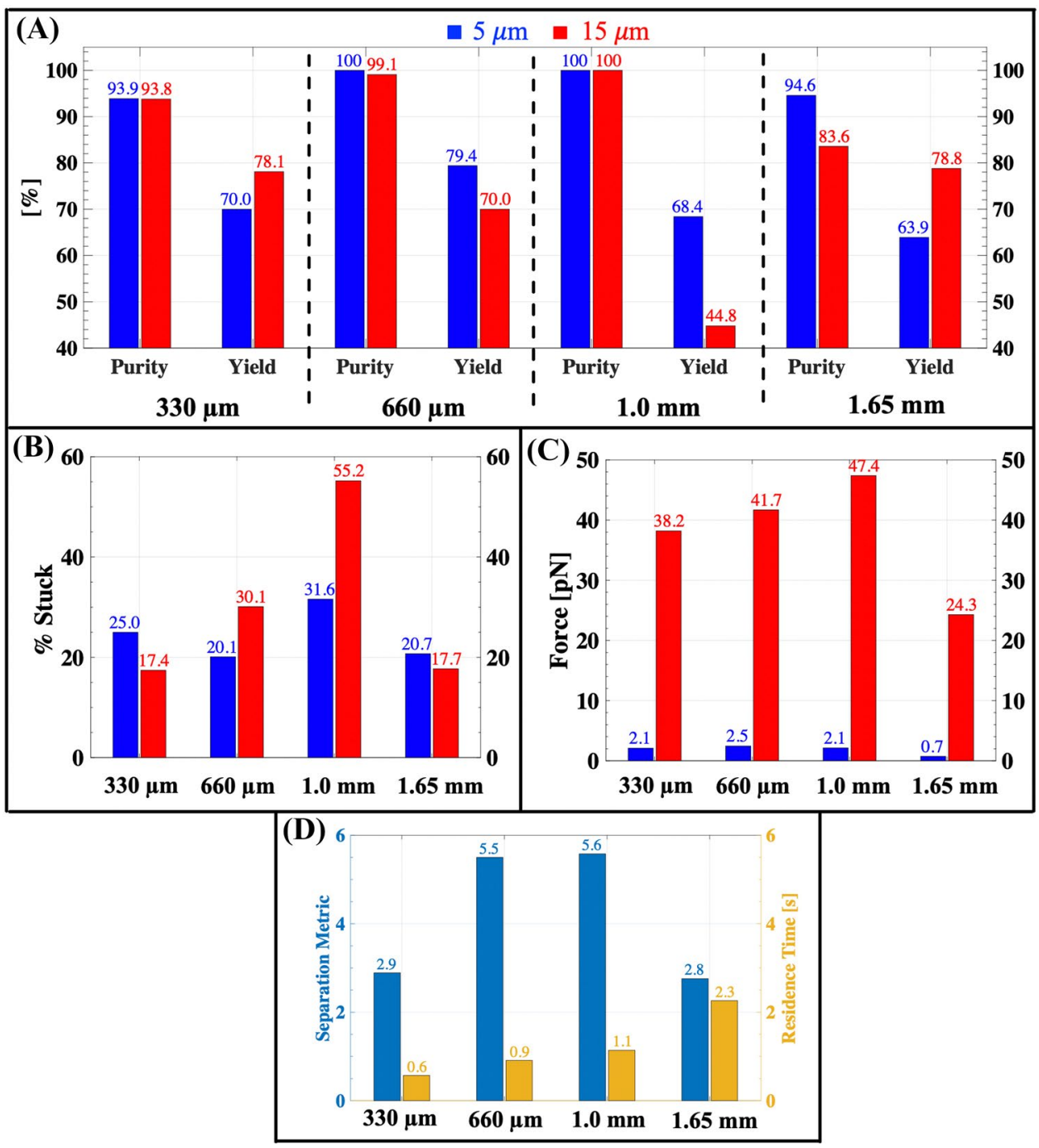

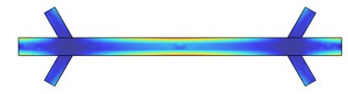

(A)

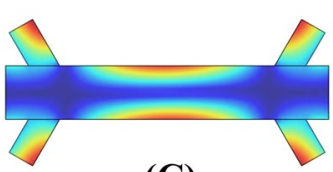

(C)

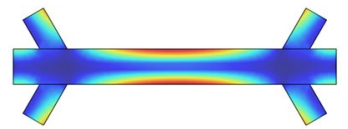

(B)

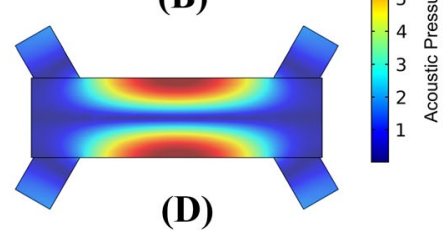

Fig. 6 Pressure distributions: a $W_{\text {chan }}=330 \mu \mathrm{m}, \quad V=90 \quad \mathrm{~V}, \quad$ b $W_{\text {chan }}=660 \mu \mathrm{m}, V=250 \mathrm{~V}, \mathbf{c} W_{\text {chan }}=1.0 \mathrm{~mm}, V=120 \mathrm{~V}, \mathbf{d}$ $W_{\text {chan }}=1.65 \mathrm{~mm}, V=16 \mathrm{~V}$

ratios of 1.0, 2.0, 3.0, 5.0 and 10. Figure 8a shows that the purity improves with increasing flow rate ratio which indicates increasing the main channel flow rate decreases the possibility of particles exiting from the unintended exit. The yield seems to increase until $Q_{\mathrm{A}} / Q_{\mathrm{B}}$ ratio becomes 5.0. There seems to be a decrease in the yield for the highest flow rate ratio of 10. It can be understood from Fig. $8 b$ that the reason for the reduction in the yield for the flow ratio of 10 is the increase in the acoustic force required to achieve separation. In all the runs of this case study, $Q_{B}$ was fixed at $20 \mu$ $\mathrm{L} / \mathrm{min}$; therefore, as the flow rate ratio is increased the main channel, the flow rate also increases which causes a need for higher acoustophoretic force. Figure 8d presents that separation metric increases as the flow rate ratio increases which is in line with the purity results obtained in Fig. 8a. It is also clear that when the main flow rate increases, residence time decreases which shows that higher throughput can be obtained with a higher separation metric. Table 2 summarizes the flow rates at the entry channels and required voltages on the piezoelectric actuators to accomplish separation. 
Fig. 7 Performance metrics at different channel depths: a purity and yield, $\mathbf{b} \%$ stuck, $\mathbf{c}$ average acoustophoretic force, $\mathbf{d}$ separation metric and residence time, e particle distribution at the main and side outlets

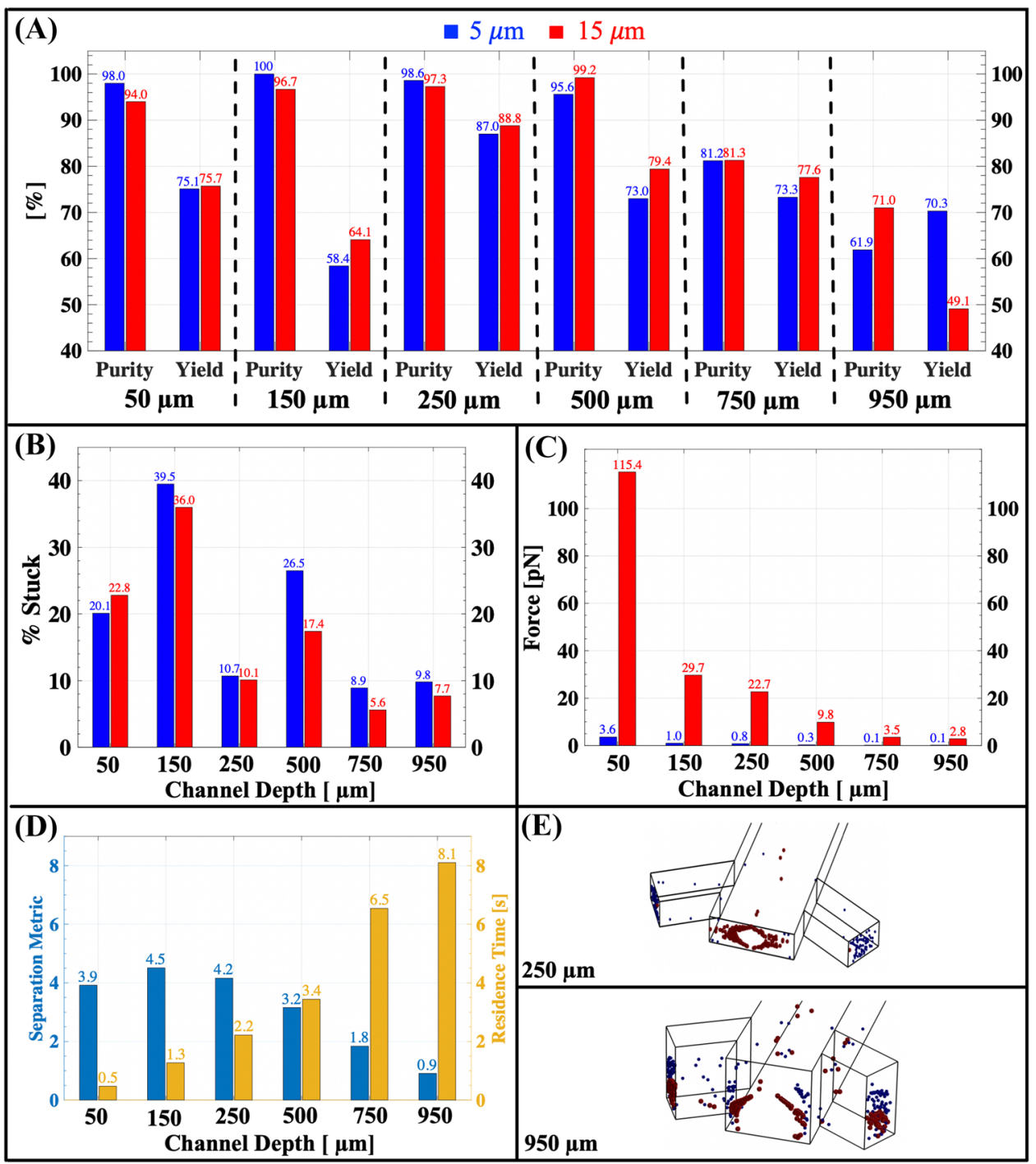

\subsection{Separation for similar size particles (case-6)}

In all previous cases, $5 \mu \mathrm{m}$ and $15 \mu \mathrm{m}$ particles are separated from each other. In Case-6, different from previous cases, the separation of two similar size particles, $5 \mu \mathrm{m}$ and $8 \mu \mathrm{m}$ particles, is simulated. Case- 6 is similar to Case- 2 in the sense that separation is performed at different channel widths and their corresponding resonance frequencies. It can be seen from Fig. 9a that the purity and yield values are significantly lower than that of Case- 2 . However, the trends in purity and yield of $5 \mu \mathrm{m}$ and $8 \mu \mathrm{m}$ particle separation is very similar to the trends in Case-2. As in Case2, separation with $2.0 \mathrm{MHz}$ resonance chip and $1.0 \mathrm{MHz}$ resonance chip is similar, and they are better compared to chips with other resonant frequencies $(700 \mathrm{kHz}$ and 400 $\mathrm{kHz}$ ). Figure $9 \mathrm{~b}$ illustrates that the percentage of the stuck particles increases as the resonance frequency of the channel decreases which is a similar behavior that of Case-2.
Figure $9 \mathrm{~d}$ depicts residence time. The behavior is again similar to that of Case-2 which is quite expected since flow rates and channel geometries are the same in both cases. Figure $9 \mathrm{~d}$ also shows that the separation metric is the highest in $1.0 \mathrm{MHz}$ resonance chip.

\subsubsection{The effect of focused particle release (case-7)}

In Case-6, it was shown that as the particle diameters of the two particle groups become closer, the performance of separation deteriorates. To improve the separation performance, the particles are released into the separation channel from a narrower region along the depth and width directions. In other words, starting positions for the particles prior to being exposed to acoustophoretic force is closer to each other. This shows the sensitivity of separation process to starting location of the particles. In Case-7, the chip with $1.0 \mathrm{~mm}$ channel width is used which has a resonant frequency near 
Fig. 8 Performance metrics at different flow rate ratios: a purity and yield, $\mathbf{b} \%$ stuck, $\mathbf{c}$ average acoustophoretic force, $\mathbf{d}$ separation metric and residence time
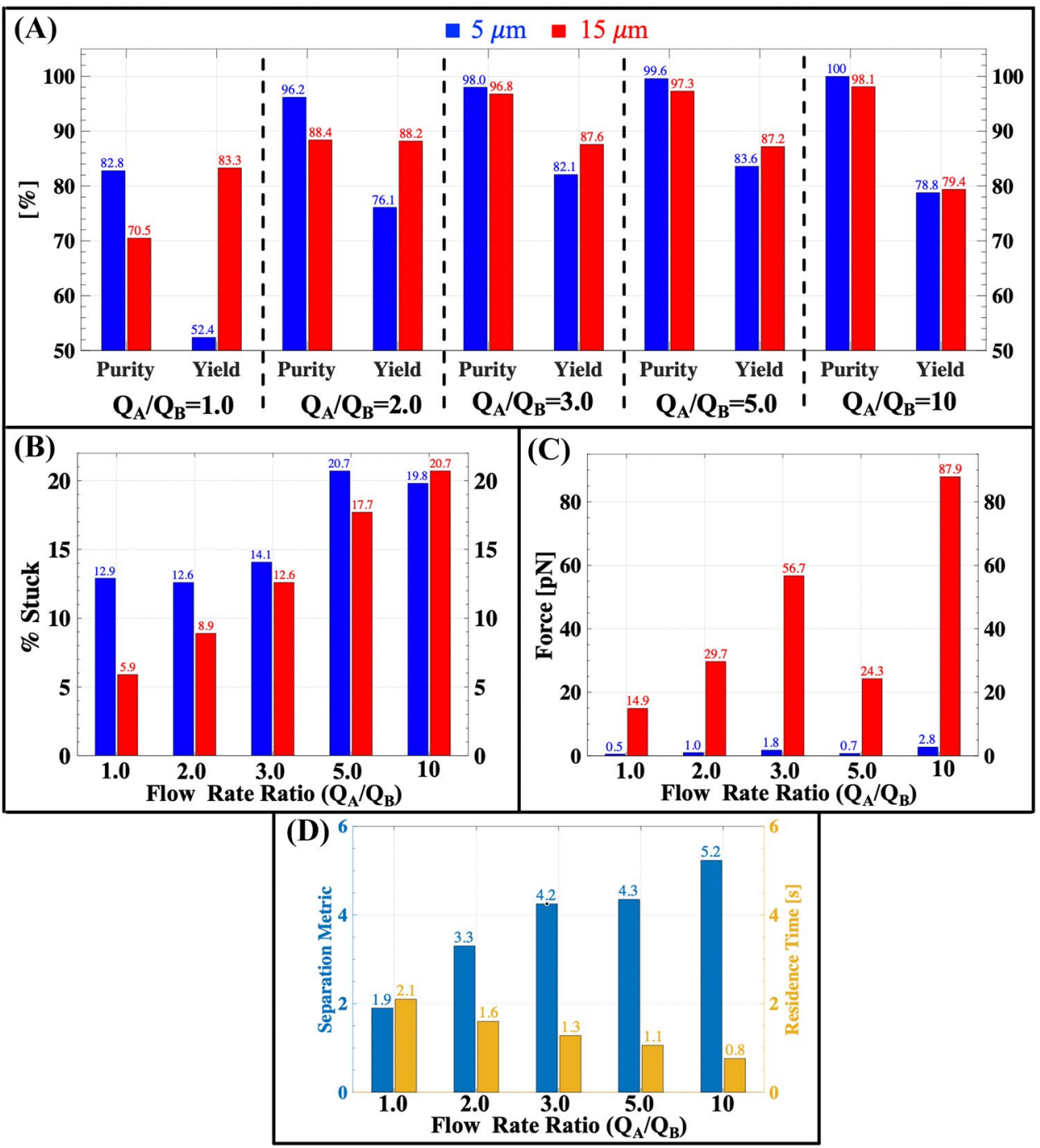

Table 2 Flow rates and voltages applied to PZT for runs with different flow rate ratio

\begin{tabular}{llll}
\hline & $\begin{array}{l}Q_{A} \\
{[\mu \mathrm{L} / \mathrm{min}]}\end{array}$ & $\begin{array}{l}Q_{B} \\
{[\mu \mathrm{L} / \mathrm{min}]}\end{array}$ & $\begin{array}{l}\text { Voltage } \\
{[\mathrm{V}]}\end{array}$ \\
\hline$Q_{\mathrm{A}} / Q_{\mathrm{B}}=1.0$ & 20 & 20 & 6 \\
$Q_{\mathrm{A}} / Q_{\mathrm{B}}=2.0$ & 40 & 20 & 9 \\
$Q_{\mathrm{A}} / Q_{\mathrm{B}}=3.0$ & 60 & 20 & 11 \\
$Q_{\mathrm{A}} / Q_{\mathrm{B}}=5.0$ & 100 & 20 & 14 \\
$Q_{\mathrm{A}} / Q_{\mathrm{B}}=10$ & 200 & 20 & 20 \\
\hline
\end{tabular}

$700 \mathrm{kHz}$. The depth of the channels is chosen to be $150 \mu \mathrm{m}$. Instead of releasing particles from randomly distributed locations along the cross-section of the channel, this time the particles are released from a narrower section with different variations. Three different variations are compared against the baseline which is the case of random distribution over the entire cross-section. The particle distributions at the inlet locations for the compared cases are demonstrated in Fig. 10. The first confined release case corresponds to releasing particles from the quarter of the width of the side channels only. The second case corresponds to releasing from the quarter of the depth of the side channel only, and the last case corresponds to releasing particles from a region which is the quarter of the width and quarter of the depth of the channel.

Figure 11a shows that narrowing of the entrance region of the particles improves the separation performance significantly. Purity and yield are significantly improved for all cases with confined release area. When the particles are released from quarter width-quarter height configuration, the purity and yield is $100 \%$. To assess the performance for a more challenging task, a size distribution is assigned for $5 \mu \mathrm{m}$ and $8 \mu \mathrm{m}$ particles. The size distributions of these two groups of diameters of particles are shown in Fig. 12a. The largest diameter of the smaller size particles is $5.8 \mu \mathrm{m}$ and the smallest diameter of the large size particles is $6.8 \mu \mathrm{m}$. It can be seen that these two diameter values are quite close 
Fig. 9 Performance metrics for similar particles: a purity and yield, b \% stuck, c average acoustophoretic force, $\mathbf{d}$ separation metric and residence time

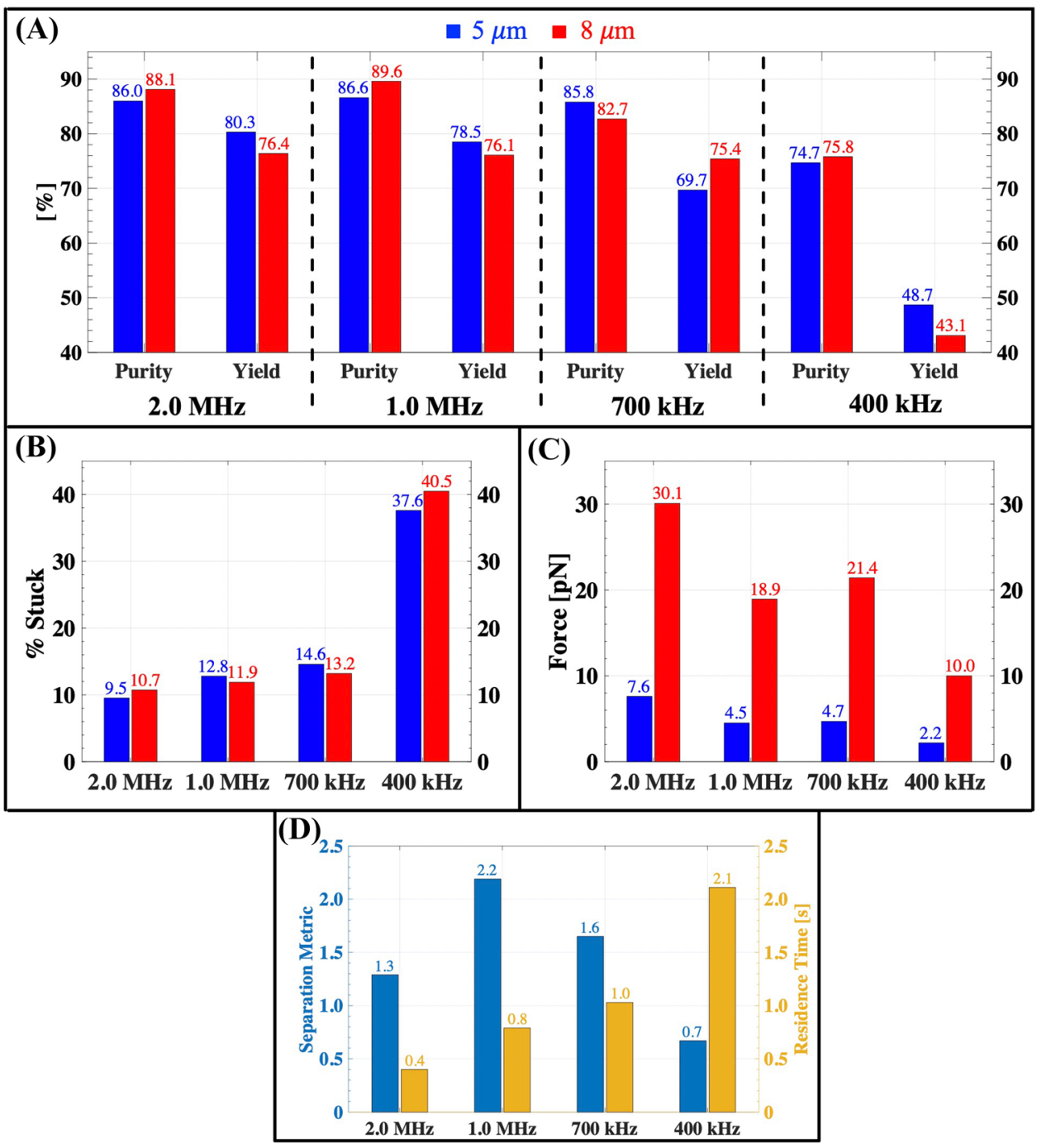

to each other and which makes acoustophoretic separation very challenging. Figure $11 \mathrm{~b}$ shows the purity and yield values for the particles with size distribution given in Fig. 12a. It can be seen that for the baseline case, the performance results are similar to single sized separation cases. On the other hand, the given size distributions challenged the focused release cases where in general the purity and yield have dropped for the particles released from quarter of the depth of the channel and quarter of the width of the channel as shown in Fig. 11b. Impressively, the case where the particles were released from a narrow section as shown in Fig. 10d, 100\% separation is still possible which shows that if the particles are released from similar initial locations, selectivity of acoustophoresis in terms of size can be quite high. Figure 11c shows that even though perfect separation is achieved in this configuration, the separation metric has significantly dropped compared to the case with no size distribution. Figure $12 \mathrm{~b}$ shows the exit locations of particles with size distribution. As seen from Fig. 11, narrowing the release region in the depth direction is more efficient than narrowing the release region in the width direction.

\section{Concluding remarks}

In this study, a 3D multiphysics numerical simulation model which couples electrical-mechanical-structural-acoustic domains is performed. The simulation results are used to calculate acoustophoretic and drag force components on particles and microparticle separation is successfully performed. The proposed simulation methodology may also be used to optimize the piezoelectric materials geometry, configuration and locations. In addition, the effects of geometry, material and electro-mechanical properties of the acoustophoretic chip components on separation performance and sensitivity of performance to these parameters can be evaluated using 
Fig. 10 Particle distribution for different focusing configurations

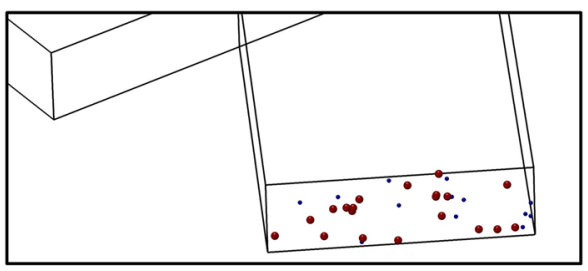

(A)

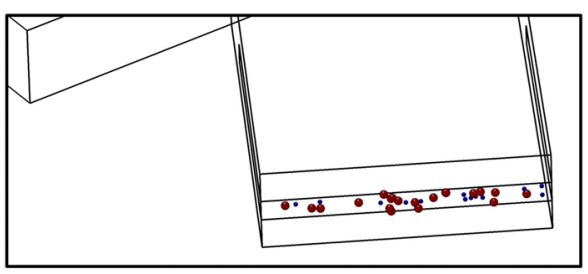

(C)

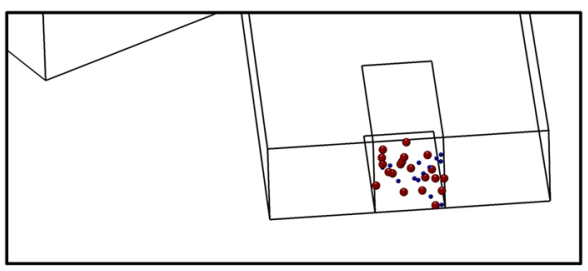

(B)

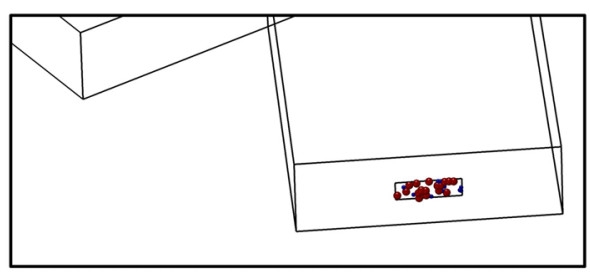

(D) the proposed numerical model. The important conclusions drawn from this study can be summarized as follows:

- Case-2 and Case-6 show that chips which are designed for higher resonance frequency (narrow width chips) resulted in higher separation performance (higher purity and yield). Moreover, the number of stuck particles increases as the resonance frequency of the chips reduces.

- Case-3 demonstrates that when the two piezoelectric actuators are driven out-of-phase, it is possible to separate microparticles at any frequency. However, if the half wavelength of the pressure wave at the frequency of excitation is quite different than the channel width, high voltage values are required to create high amplitude standing pressure wave in the channel. Even though it is possible to separate microparticles at every frequency theoretically; you need to operate the microfluidic device at a frequency where the half wavelength of the pressure wave needs to be close to channel width of the separation channel practically (which is the common practice in the acoustofluidic literature), since the required input levels become too high if the half wavelength condition is not satisfied.

- Case-4 indicates that separation at shallow channels can be difficult since the flow velocity will be too high in the separation channel for a given flow rate. Due to high flow velocity, high acoustophoretic forces therefore high voltages are needed on the PZTs and this resulted in high number of stuck particles at the side entry inlets of the channel.

- Case-4 also shows that separation at deep channels (where the pressure wavelength is close to channel depth) results in variation of pressure field along the height direction. Therefore, at certain height levels, the pressure field generates enough acoustophoretic forces to accomplish separation. On the other hand, at some other height levels, pressure field is not high enough for separation. If voltage is further increased to achieve separation at the weak pressure height level, it results in high acoustic pressures at another level where the small diameter microparticles start to exit from main channel (unintended channel). Therefore, the channel should not be at depths that are comparable to pressure wavelength.

- Case-5 shows that as the ratio of the main channel flow rate to side channel increases, the purity and yield of microparticle separation significantly improves. On the other hand, if the flow velocity becomes very fast, high voltages on the PZTs will be required for separation. Main channel flow rate which drags the particles towards the walls seems to improve the purity at the channel exits but if the main channel flow rate is too high, the required acoustophoretic force becomes too large and the particles close to the walls get stuck at the side walls as well as the top and bottom walls due to the increased acoustophoretic force.

- Case-6 shows that as the particle sizes get closer to each other, separation performance drops. In Case-7, to improve the separation, particles are released from a narrower region which significantly improved the separation quality. Forcing the particles into a narrower region before entering the separation channel is an approach which can be found in the literature (Tenje et al. 2015) (the process was called pre-alignment in that study, we prefer to call focusing). Similar to our findings, it was stated that pre-alignment improved the separation performance significantly. In the same case study, size distribution is given for the small and large diameter particle groups to further understand the limits of size-based separation capability of acoustophoretic process. The existence of size distribution decreased purity and yield values of the quarter width and quarter height release cases. 
Fig. 11 Performance metrics for similar particles at different focusing configurations: a purity and yield (without size distribution), b purity and yield (with size distribution), c separation metric and residence time with and without size distribution
Fig. 12 Particle distributions for $5 \mu \mathrm{m}$ and $8 \mu \mathrm{m}$ particles: a size distribution at the inlet of the channel, b particle distribution at the outlets

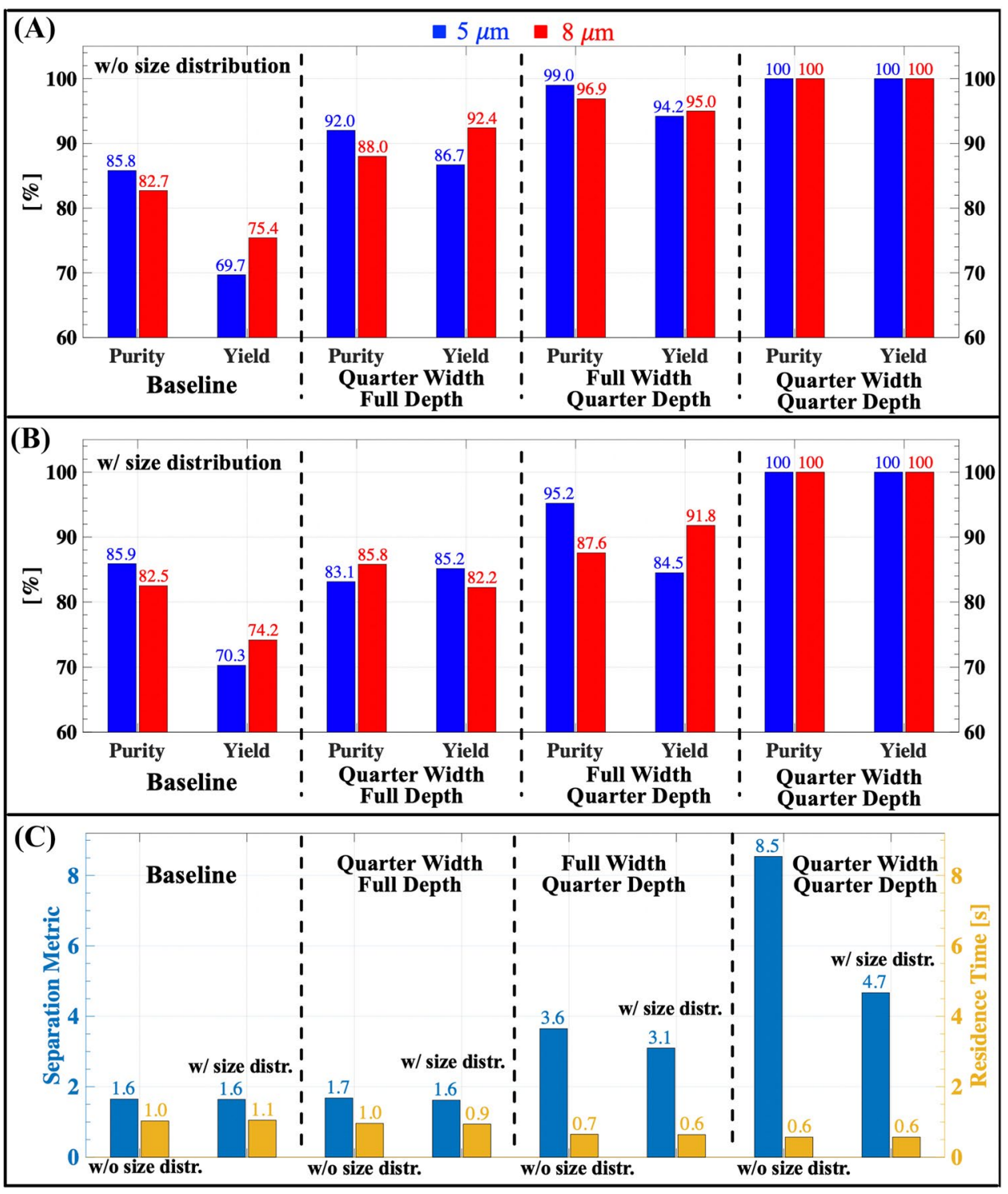


It is observed that confining the particle release along the height direction results in better separation performance compared to confined particle release along the width dimension. Even for the particles with size distribution, when the particles are released from the narrowest area (the quarter of the width and the quarter of the height of the side channel cross section) perfect separation was achieved. This case study shows that if particles can be focused prior to separation, acoustophoresis will have a remarkable resolution in size-based separation.

Acknowledgements This study is financially supported by the Turkish Scientific and Technical Research Council, under Grant No. 115M684. B.Ç. would like to acknowledge fundings from the Turkish Academy of Sciences through Outstanding Young Scientist Program (TÜBAGEBIP) and Science Academy Distinguished Young Scientist Award (BAGEP).

\section{References}

Ahmed D, Ozcelik A, Bojanala N, Nama N, Upadhyay A, Chen Y, Hanna-Rose W, Huang TJ (2016) Rotational manipulation of single cells and organisms using acoustic waves. Nature Communications 7:11085 EP 03

Antfolk M, Muller PB, Augustsson P, Bruus H, Laurell T (2014) Focusing of sub-micrometer particles and bacteria enabled by two-dimensional acoustophoresis. Lab Chip 14:2791-2799

Antfolk Maria, Kim Soo Hyeon, Koizumi Saori, Fujii Teruo, Laurell Thomas (2017) Label-free single-cell separation and imaging of cancer cells using an integrated microfluidic system. Scientific Reports 7:46507 EP

Baasch T, Dual J (2018) Acoustofluidic particle dynamics: beyond the rayleigh limit. J Acoust Soc Am 143(1):509-519

Baasch T, Leibacher I, Dual J (2017) Multibody dynamics in acoustophoresis. J Acoust Soc Am 141(3):1664-1674

Bruus H (2011) Acoustofluidics 1: governing equations in microfluidics. Lab Chip 11:3742-3751

Bruus H (2012) Acoustofluidics 2: perturbation theory and ultrasound resonance modes. Lab Chip 12:20-28

Bruus H (2012) Acoustofluidics 7: the acoustic radiation force on small particles. Lab Chip 12:1014-1021

Buyukkocak S, Ozer MB, Cetin B (2014) Numerical modeling of ultrasonic particle manipulation for microfluidic applications. Microfluid Nanofluid 17(6):1025-1037

Cai F, Meng L, Jiang C, Pan Y, Zheng H (2010) Computation of the acoustic radiation force using the finite-difference time-domain method. J Acoust Soc Am 128(4):1617-1622

Cetin B, Ozer MB, Solmaz ME (2014) Microfluidic bio-particle manipulation for biotechnology. Biochem Eng J 92:63-82

Cetin Barbaros, Ozer M Bulent, Cagatay Erdem, Buyukkocak Suleyman (2016) An integrated acoustic and dielectrophoretic particle manipulation in a microfluidic device for particle wash and separation fabricated by mechanical machining. Biomicrofluidics 10(014112)

Cetin Barbaros, Oner S Dogan, Baranoglu Besim (2017) Modeling of dielectrophoretic particle motion: Point particle vs finite-sized particle. Electrophoresis 38:1407-1418

Ceylan H, Giltinan J, Kozielski K, Sitti M (2017) Mobile microrobots for bioengineering applications. Lab Chip 17:1705-1724
Cushing K, Undvall E, Ceder Y, Lilja H, Laurell T (2018) Reducing WBC background in cancer cell separation products by negative acoustic contrast particle immuno-acoustophoresis. Anal Chim Acta 1000:256-264

Dow P, Kotz K, Gruszka S, Holder J, Fiering J (2018) Acoustic separation in plastic microfluidics for rapid detection of bacteria in blood using engineered bacteriophage. Lab Chip 18:923-932

Garbin A, Leibacher I, Hahn P, Le Ferrand H, Studart A, Dual J (2015) Acoustophoresis of disk-shaped microparticles: a numerical and experimental study of acoustic radiation forces and torques. $\mathbf{J}$ Acoust Soc Am 138(5):2759-2769

Garofalo F (2019) On the methodologies for the assessment of the impact of parameters in acoustophoretic separation devices. Microfluid Nanofluid 23(7):90

Glynne-Jones P, Mishra PP, Boltryk RJ, Hill M (2013) Efficient finite element modeling of radiation forces on elastic particles of arbitrary size and geometry. J Acoust Soc Am 133(4):1885-1893

Gorkov LP (1962) On the forces acting on a small particle in an acoustic field in an ideal fluid. Dokl Akad Nauk SSSR 6:773-776

Gralinski I, Alan T, Neild A (2012) Non-contact acoustic trapping in circular cross-section glass capillaries: a numerical study. J Acoust Soc Am 132(5):2978-2987

Gu Y, Chen C, Wang Z, Huang P-H, Fu H, Wang L, Wu M, Chen Y, Gao T, Gong J, Kwun J, Arepally GM, Huang TJ (2019) Plasticbased acoustofluidic devices for high-throughput, biocompatible platelet separation. Lab Chip 19:394-402

Hahn P, Leibacher I, Baasch T, Dual J (2015) Numerical simulation of acoustofluidic manipulation by radiation forces and acoustic streaming for complex particles. Lab Chip 15:4302-4313

Hahn P, Schwab O, Dual J (2014) Modeling and optimization of acoustofluidic micro-devices. Lab Chip 14:3937-3948

Karlsen JT, Bruus H (2015) Forces acting on a small particle in an acoustical field in a thermoviscous fluid. Phys Rev E 92:043010

Kaynak M, Ozcelik A, Nourhani A, Lammert PE, Crespi VH, Huang TJ (2017) Acoustic actuation of bioinspired microswimmers. Lab Chip 17:395-400

King Louis V (1934) On the acoustic radiation pressure on spheres. Proc R Soc Lond Ser A Math Phys Sci 147(861):212-240

Laurell T, Petersson F, Nilsson A (2007) Chip integrated strategies for acoustic separation and manipulation of cells and particles. Chem Soc Rev 36:492-506

Lei J, Hill M, Glynne-Jones P (2014) Numerical simulation of 3d boundary-driven acoustic streaming in microfluidic devices. Lab Chip 14:532-541

Lissandrello C, Dubay R, Kotz KT, Fiering J (2018) Purification of lymphocytes by acoustic separation in plastic microchannels. SLAS Technol Transl Life Sci Innovat 23(4):352-363

Liu Z, Kim Y-J, Wang H, Han A (2016) Effects of fluid medium flow and spatial temperature variation on acoustophoretic motion of microparticles in microfluidic channels. J Acoust Soc Am 139(1):332-349

Magnusson C, Augustsson P, Lenshof A, Ceder Y, Laurell T, Lilja H (2017) Clinical-scale cell-surface-marker independent acoustic microfluidic enrichment of tumor cells from blood. Anal Chem 89(22):11954-11961

Mao Z, Xie Y, Guo F, Ren L, Huang P-H, Chen Y, Rufo J, Costanzo F, Huang TJ (2016) Experimental and numerical studies on standing surface acoustic wave microfluidics. Lab Chip 16:515-524

Mengxi W, Mao Z, Chen K, Bachman H, Chen Y, Rufo J, Ren L, Li P, Wang L, Huang TJ (2017) Acoustic separation of nanoparticles in continuous flow. Adv Funct Mater 27(14):1606039

Muller PB, Barnkob R, Jensen MJH, Jakob Herring M, Bruus H (2012) A numerical study of microparticle acoustophoresis driven by acoustic radiation forces and streaming-induced drag forces. Lab Chip 12:4617-4627 
Muller PB, Rossi M, Marín AG, Barnkob R, Augustsson P, Laurell T, Kähler CJ, Bruus H (2013) Ultrasound-induced acoustophoretic motion of microparticles in three dimensions. Phys Rev E 88:023006

Nama N, Barnkob R, Mao Z, Khler CJ, Costanzo F, Huang TJ (2015) Numerical study of acoustophoretic motion of particles in a pdms microchannel driven by surface acoustic waves. Lab Chip 15:2700-2709

Ohlsson P, Petersson K, Augustsson P, Laurell T (2018) Acoustic impedance matched buffers enable separation of bacteria from blood cells at high cell concentrations. Sci Rep 8(1):9156

Petersson K, Jakobsson O, Ohlsson P, Augustsson P, Scheding S, Malm J, Laurell T (2018) Acoustofluidic hematocrit determination. Anal Chim Acta 1000:199-204

Sehgal P, Kirby BJ (2017) Separation of 300 and $100 \mathrm{~nm}$ particles in fabry-perot acoustofluidic resonators. Anal Chem 89(22):12192-12200

Settnes M, Bruus H (2012) Forces acting on a small particle in an acoustical field in a viscous fluid. Phys Rev E 85:016327

Silva GT, Bruus H (2014) Acoustic interaction forces between small particles in an ideal fluid. Phys Rev E 90:063007

Silva R, Dow P, Dubay R, Lissandrello C, Holder J, Densmore D, Fiering J (2017) Rapid prototyping and parametric optimization of plastic acoustofluidic devices for blood-bacteria separation. Biomed Microdev 19(3):70

Tenje M, Lundgren MN, Swärd-Nilsson A-M, Kjeldsen-Kragh J, Lyxe L, Lenshof A (2015) Acoustophoretic removal of proteins from blood components. Biomed Microdev 17(5):95

Trippa G, Ventikos Y, Taggart DP, Coussios C-C (2011) Cfd modeling of an ultrasonic separator for the removal of lipid particles from pericardial suction blood. IEEE Trans Biomed Eng 58(2):282-290

Trujillo FJ, Eberhardt S, Muller D, Dual J, Knoerzer K (2013) Multiphysics modelling of the separation of suspended particles via frequency ramping of ultrasonic standing waves. Ultrason Sonochem 20(2):655-666

Ung WL, Mutafopulos K, Spink P, Rambach RW, Franke T, Weitz DA (2017) Enhanced surface acoustic wave cell sorting by $3 d$ microfluidic-chip design. Lab Chip 17:4059-4069

Urbansky A, Ohlsson P, Lenshof A, Garofalo F, Scheding S, Laurell T (2017) Rapid and effective enrichment of mononuclear cells from blood using acoustophoresis. Sci Rep 7(1):17161

Vitali V, Yang T, Minzioni P (2018) Separation efficiency maximization in acoustofluidic systems: study of the sample launch-position. RSC Adv 8:38955-38964

Wang J, Dual J (2009) Numerical simulations for the time-averaged acoustic forces acting on rigid cylinders in ideal and viscous fluids. J Phys A Math Theor 42(28):285502

Wang J, Dual J (2012) Theoretical and numerical calculation of the acoustic radiation force acting on a circular rigid cylinder near a flat wall in a standing wave excitation in an ideal fluid. Ultrasonics 52(2):325-332

Yang T, Vitali V, Minzioni P (2018) Acoustofluidic separation: impact of microfluidic system design and of sample properties. Microfluid Nanofluid 22(4):44

Yosioka K, Kawasima Y (1955) Acoustic radiation pressure on a compressible sphere. Acoustica 5:167-173

Zhou W, Wang J, Wang K, Huang B, Niu L, Li F, Cai F, Chen Y, Liu X, Zhang X, Cheng H, Kang L, Meng L, Zheng H (2017) Ultrasound neuro-modulation chip: activation of sensory neurons in caenorhabditis elegans by surface acoustic waves. Lab Chip $17: 1725-1731$

Publisher's Note Springer Nature remains neutral with regard to jurisdictional claims in published maps and institutional affiliations. 